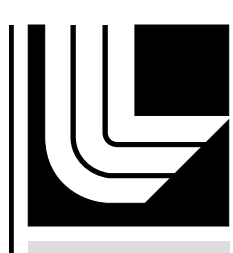

LA W RENCE LIVERM ORE NATIONAL LABORATORY

\title{
An IR-Selected Galaxy Cluster at $\mathrm{Z}=1.41$
}

S. A. Stanford, P. R. Eisenhardt, M. Brodwin, A. H. Gonzalez, D. Stern, B. Jannuzi, A. Dey, M. J. I. Brown, E. McKenzie, R. Elston

April 26, 2006

The Astrophysical Journal Letters 
This document was prepared as an account of work sponsored by an agency of the United States Government. Neither the United States Government nor the University of California nor any of their employees, makes any warranty, express or implied, or assumes any legal liability or responsibility for the accuracy, completeness, or usefulness of any information, apparatus, product, or process disclosed, or represents that its use would not infringe privately owned rights. Reference herein to any specific commercial product, process, or service by trade name, trademark, manufacturer, or otherwise, does not necessarily constitute or imply its endorsement, recommendation, or favoring by the United States Government or the University of California. The views and opinions of authors expressed herein do not necessarily state or reflect those of the United States Government or the University of California, and shall not be used for advertising or product endorsement purposes. 


\title{
AN IR-SELECTED GALAXY CLUSTER AT $Z=1.41$
}

\author{
S.A. Stanford ${ }^{1,2}$, Peter R. Eisenhardt ${ }^{3}$, Mark Brodwin ${ }^{3}$, Anthony H. Gonzalez ${ }^{4}$, Daniel Stern ${ }^{3}$, Buell $^{2}$ \\ Jannuzi $^{5}$, Arjun Dey ${ }^{5}$, Michael J. I. Brown ${ }^{6}$, Eric McKenzie ${ }^{4}$, And Richard Elston ${ }^{4,7}$ \\ Draft version February 21, 2006
}

\begin{abstract}
We report the discovery of a galaxy cluster at $z=1.41$. ISCS J143809+341419 was found in the Spitzer/IRAC Shallow Survey of the Boötes field in the NOAO Deep Wide-Field Survey carried out by IRAC. The cluster candidate was initially identified as a high density region of objects with photometric redshifts in the range $1.3<z<1.5$. Optical spectroscopy of a limited number of objects in the region shows that 5 galaxies within a $\sim 120 \operatorname{arcsec}$ diameter region lie at $z=1.41 \pm$ 0.01. Most of these member galaxies have broad-band colors consistent with the expected spectral energy distribution of a passively-evolving elliptical galaxy formed at high redshift. The redshift of ISCS J143809+341419 is the highest currently known for a spectroscopically-confirmed cluster of galaxies.
\end{abstract}

Subject headings: galaxies: clusters — galaxies: evolution — galaxies: formation

\section{INTRODUCTION}

High-redshift galaxy clusters provide important tools in the study of galaxy formation and evolution. The galaxy populations of rich cluster cores at $z>1$ tend to be dominated by massive ellipticals (e.g. Stanford et al. 1997; Rosati et al. 1999; Stanford et al. 2002; van Dokkum \& Stanford 2003; Blakeslee et al. 2003; Rosati et al. 2004; Postman et al. 2005; Mullis et al. 2005; Mei et al. 2005), which are useful probes of galaxy evolution because their stellar populations appear to be relatively simple. As the highest overdensity regions, clusters should contain the oldest (and most massive) galaxies, so that by probing $z>1$ we can determine useful constraints on the galaxy formation process. The connection between such stellar populations and the ellipticals in present-epoch clusters (Bower et al. 1992) has been the subject of extensive analysis in recent years (e.g. Stanford, Eisenhardt, \& Dickinson 1998; van Dokkum et al. 1998; Lubin et al. 1998; De Propris et al. 1999; Kelson et al. 2000). The nature of elliptical galaxy formation in clusters at $z>1$ depends strongly on the importance and mode of merging in assembling the stellar mass (van Dokkum \& Franx 2001; Conselice 2005). Beyond $z \sim 1$ in cosmologically-flat CDM models, the amount of merging occurring within the prior $\sim 1$ Gyr is large and should seriously inflate the locus of early-type galaxy colors due to interaction-induced starbursts (Kauffmann 1996), although evidence exists for "dry" mergers that could alleviate this issue (van Dokkum 2005). On the other hand,

\footnotetext{
1 University of California, Davis, CA 95616; adam@igpp.ucllnl.org

2 Institute of Geophysics and Planetary Physics, Lawrence Livermore National Laboratory, Livermore, CA 94551

3 Jet Propulsion Laboratory, California Institute of Technology, Pasadena, CA 91109; prme@kromos.jpl.nasa.gov; Mark.Brodwin@jpl.nasa.gov; stern@thisvi.jpl.nasa.gov

4 Department of Astronomy, University of Florida, Gainesville, FL 32611; anthony@astro.ufl.edu; eric@astro.ufl.edu

5 National Optical Astronomy Observatories Tucson, AZ 85726-6732; jannuzi@noao.edu; dey@noao.edu

6 Department of Astrophysical Sciences, Peyton Hall, Princeton University, Princeton, NJ 08544; mbrown@astro.princeton.edu

7 Deceased
}

models in which ellipticals formed by a monolithic collapse at high- $z$ predict a tight color-magnitude relation out to at least $z \sim 2$ for reasonable cosmologies (Eggen, Lynden-Bell, \& Sandage 1962). The identification of clusters at $z>1$ and the characterization of their galaxy populations provides a powerful means of testing elliptical galaxy formation theories.

One proven method of finding $z>1$ cluster candidates is deep infrared sky surveys. Because they are massive and their rest-frame light peaks at $1-2 \mu \mathrm{m}$, early-type galaxies stand out from the background in the observedframe near- and mid-IR light out to $z \sim 2$. Therefore, to the extent that galaxy clusters are composed of galaxies, near- and mid-IR imaging surveys offer a viable alternative to $\mathrm{X}$-ray searches for very high redshift clusters. Both methods need to be exploited to sample the entire range of galaxy clusters in the key $1<z<2$ epoch because it is unclear when and how massive galaxies and the associated intracluster medium (ICM) are formed. One advantage of using infrared imaging surveys is that, in addition to identification of massive clusters with an ICM that would be detected by an X-ray survey, they can be extended to probe to lower mass scales and identify the building blocks that yield massive clusters at lower redshift. We describe here the discovery of a $z>1$ cluster which resulted from an imaging survey conducted by the InfraRed Array Camera (IRAC; Fazio et al. 2004) onboard the Spitzer Space Telescope (Werner et al. 2004). Except where noted, the assumed cosmological parameters are $\mathrm{H}_{0}=70 \mathrm{~km} \mathrm{~s}^{-1} \mathrm{Mpc}^{-1}, \Omega_{m}=0.3$ and $\Lambda=0.7$.

\section{OBSERVATIONS \\ 2.1. Sample Definition}

To identify galaxy cluster candidates in the Boötes field we made use of the IRAC Shallow Survey (Eisenhardt et al. 2004), the FLAMEX Survey, which provides $J K_{s}$ imaging down to $K_{s}=19.2$ (R. Elston et al. in preparation), and the NOAO Deep Wide-Field Survey (NDWFS), which provides deep $B_{w} R I$ imaging (Januzzi \& Dey 1999). A catalog was defined using the $4.5 \mu \mathrm{m}$ data from the IRAC survey. Photometric redshifts were estimated from matched photometry in the $B_{w} R I J K$ and 


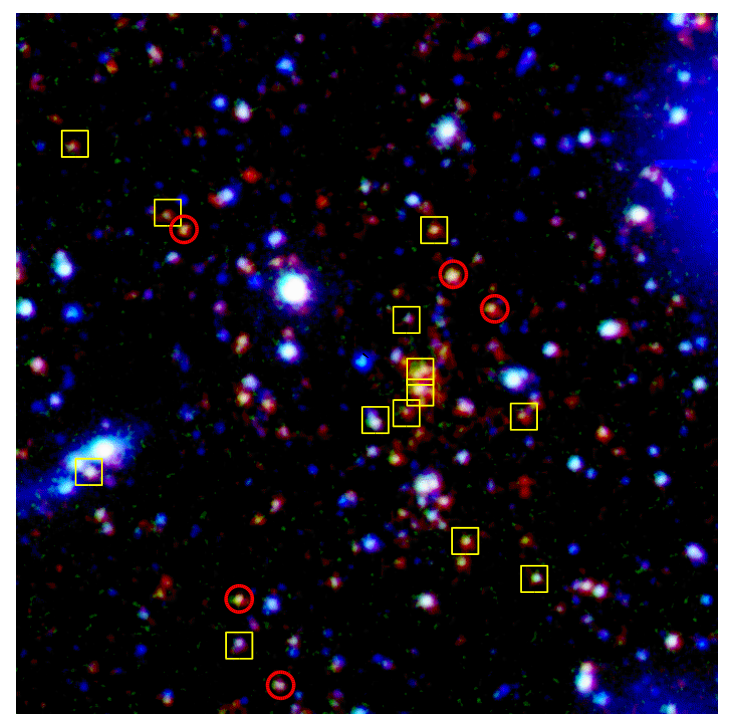

FIG. $1 .-I, K, 4.5 \mu \mathrm{m}$ false-color image of ISCS J143809+341419 covering $3^{\prime}$ on a side. Objects with photometric redshifts at $1.25<$ $z<1.55$ are marked by the yellow boxes. Photometric redshifts were not calculated for some of the fainter objects in the $4.5 \mu \mathrm{m}$ catalog so some objects visible in the image which are not marked with boxes in fact may be in the cluster. The 5 spectroscopically confirmed member galaxies are marked by the red circles. North is up and East is to the left in the image.

the IRAC 3.6 and $4.5 \mu \mathrm{m}$ bands. Then a wavelet search was carried out using the photometric redshift probability distributions to detect structures on physical scales of $200<r<800 \mathrm{kpc}$. Bootstrap simulations were then used to quantify the significance of each detection. The details of the sample selection and identification of cluster candidates are given in P. Eisenhardt et al. (in preparation), and of the photometric redshift process in $\mathrm{M}$. Brodwin et al. (in preparation). We report here on the candidate cluster ISCS J143809+341419, shown in Figure 1. The prefix ISCS stands for the IRAC Shallow survey Cluster Search. The cluster overdensity relative to that of similar size areas in the entire Boötes field in the IRAC Shallow Survey is shown in Figure 2.

\subsection{Keck Spectroscopy}

In order to make sure that the candidate cluster is not a chance alignment along the line of sight, we obtained optical spectroscopy of objects in ISCS J143809+341419. Objects were selected based on their photometric redshifts. The $I$-band magnitudes of the primary galaxy targets were $22.0<I<24.3$. We prepared a slitmask including slitlets for 14 objects with photometric redshifts $1.25<z_{p}<1.55$ within 3 arcmin of the nominal cluster center. The range in $z_{p}$ was chosen to cover the nominal photometric redshift of the cluster $\pm 1 \sigma$. Two other objects in the $4.5 \mu \mathrm{m}$ catalog were included so as to fill out the mask. The slits had widths of 1.3 arcsec and minimum lengths of 10 arcsec. The slitmask was used with the Low Resolution Imaging Spectrograph (Oke et al. 1995) on the $10 \mathrm{~m}$ Keck II telescope on UT 2005 June 04 to obtain deep spectroscopy. On the red side,

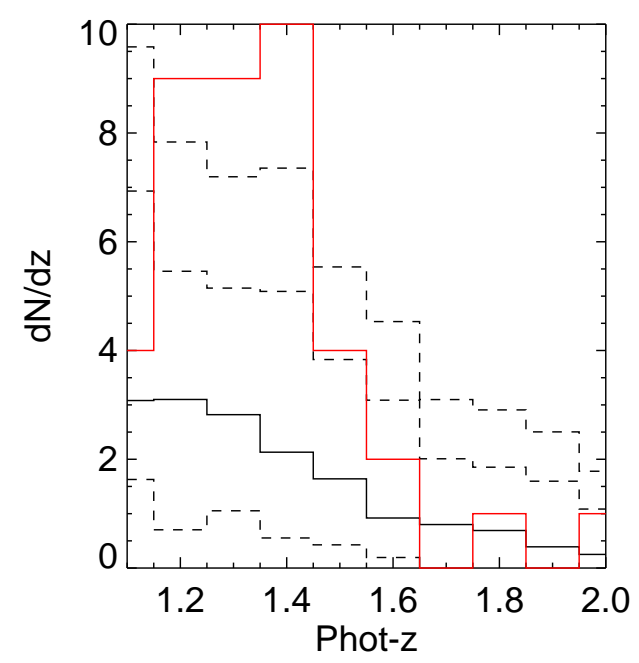

FIG. 2.- Histogram vs redshift showing the photometric redshifts of galaxies in the IRAC Shallow Survey. The area covered by the solid red histogram is $3^{\prime} \times 3^{\prime}$ around the $z=1.41$ cluster. The solid black histogram represents the average of $1003^{\prime} \times 3^{\prime}$ patches randomly chosen from the entire $\sim 9$ degree $^{2}$ IRAC survey in the Boötes field. The dashed histograms represent the 1 and 2 $\sigma$ uncertainties on the average value histogram.

we used the $400 \mathrm{l} \mathrm{mm}^{-1}$ grating, which is blazed at 8500 $\AA$, to cover a nominal wavelength range of 6000 to 9800 $\AA$, depending on the position of a slit in the mask. The dispersion of $\sim 1.8 \AA$ pixel $^{-1}$ resulted in a spectral resolution of $R \sim 900$. On the blue side we used the 400 line grism which is blazed at $3400 \AA$ and provides coverage from the atmospheric cutoff up to $\sim 5800 \AA$ where the dichroic splits the light between the two sides of LRIS. We obtained $7 \times 1800 \mathrm{~s}$ exposures with this setup in photometric conditions with 0.9 arcsec seeing. Objects were shifted along the long axis of the slits between exposures to enable better sky subtraction and fringe correction. The observations were carried out with the slitlets aligned close to the parallactic angle.

The slitmask data were separated into individual spectra and then reduced using standard longslit techniques. A relative flux calibration was obtained from longslit observations of the standard stars HZ 44 and Wolf 1346. One-dimensional spectra were extracted from the sum of all the reduced data for each of the 16 slitlets for both the red and blue sides. For the targets in the cluster, only the red side data are useful and the blue side data will not be discussed further.

\section{RESULTS}

\subsection{Optical Spectroscopy}

Spectra were obtained for 9 of the 14 photometricallyselected galaxies with quality sufficient for determining the redshifts; the other 5 were deemed too faint for reliable identification of spectral features. Only one of these 9 objects has a redshift outside of the photometric redshift range. Four objects have redshifts within the range $1.4147<z_{s}<1.4172$ so are considered to be members of a cluster. A fifth object has $z_{s}=1.4028$ which is somewhat lower than the redshifts of the other four but still likely to be a cluster member. A histogram 


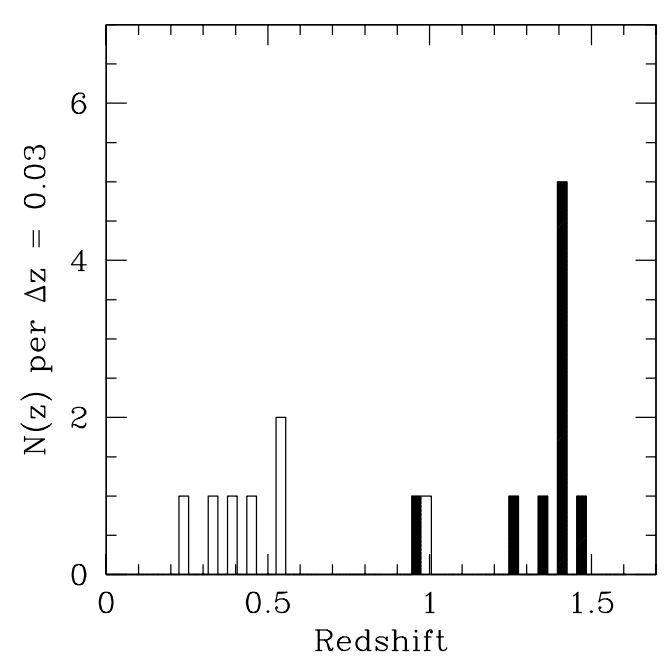

FIG. 3.- Histogram of all redshifts determined in the Keck/LRIS slitmask observation. The shaded objects are those with photometric redshifts $1.25<z_{p}<1.55$.

of all redshifts determined from the mask (including 5 serendipitous sources) is shown in Figure 3. The spectroscopy validates our photometric redshifts in the range $1.3<z<1.5$ (M. Brodwin et al. in preparation).

The spectroscopic members are listed in Table 1. The table gives the Vega magnitude [4.5] in the IRAC $4.5 \mu \mathrm{m}$ band, the photometric and spectroscopic redshifts, and the template number of the best-fit as determined by the photometric redshift estimator. These template numbers are from Brodwin (2004) based on the spectral templates of Coleman, Wu, \& Weedman (1980). The approximate correspondence to galaxy type spectra is 0 for elliptical, 7 for an Sbc, and 13 for an Scd. In line with the template fitting, the optical spectra of most of the members show absorption lines (Ca II H+K, $\lambda 3830, \mathrm{MgI} \lambda 2852$ and MgII $\lambda 2800$ ) and spectral breaks (D4000, B3260, B2900) similar to those of present-epoch ellipticals. The spectra of the member galaxies are shown in Figure 4. Only one of the 5 members,IRAC J143811+341256, has a prominent emission line; the photometry of this same object was fit best by a relatively late-type template. To better determine the redshifts of the 4 early-type cluster members, we used the Fourier quotient technique as implemented in the FXCOR task of IRAF. The red members were cross-correlated with an early-type galaxy template (Kinney et al. 1996). The resulting redshifts, along with their formal uncertainties, are listed in Table 1 . We adopt a nominal center for the cluster of $\alpha=14^{h} 38^{m} 09^{\mathrm{s}} 4, \delta=+34^{\circ} 14^{\prime} 19^{\prime \prime}$ (J2000), the centroid of the wavelet detection, and a nominal redshift $z=1.413$. Given the small number of members it is premature to estimate the velocity dispersion of the galaxies.

\subsection{Optical and Infrared Photometry}

A color-magnitude diagram for all objects in a $3^{\prime}$ area at the cluster are shown in Figure 5 . The objects with photometric redshifts in the range $1.25<z<1.55$ are shown by the red diamonds, and those with spectroscopic redshifts $1.40<z<1.42$ are shown by the blue squares.

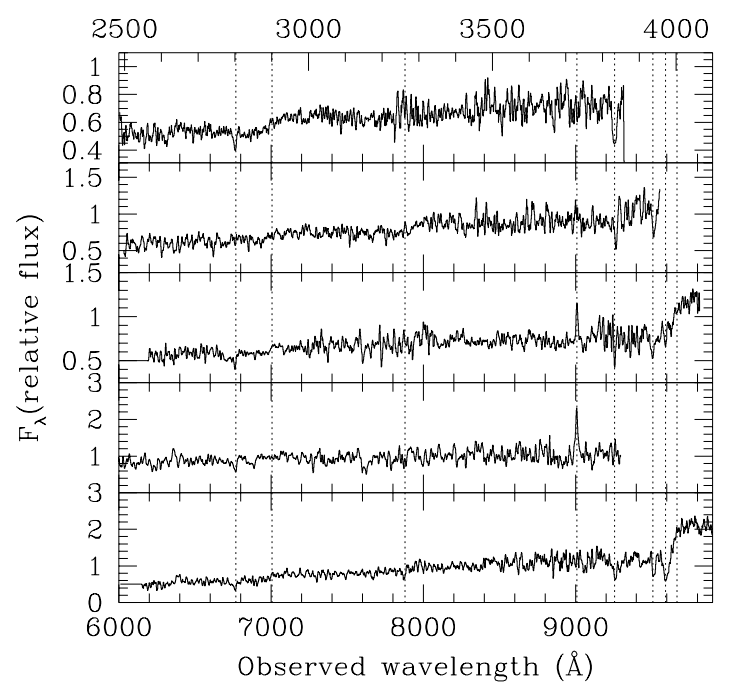

FIG. 4.- Optical spectra of the 5 member galaxies after being smoothed by an 5 pixel boxcar. The following spectral features (though not present in every object) are marked in increasing wavelength by the dotted lines: Mg II $\lambda 2800$, B2900, B3260, [OII] $\lambda 3727$, $\mathrm{Mg}$ I $\lambda 3830$, Ca II K and H, and D4000. The rest frame wavelength at $z=1.4166$ is shown along the top.

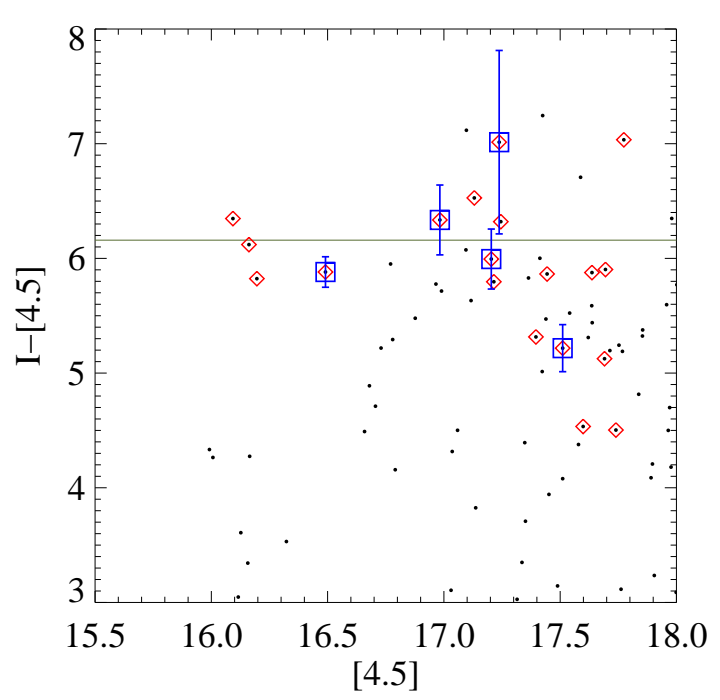

FIG. 5.- Color-magnitude diagram of the cluster in $I-[4.5]$ vs [4.5]. The magnitudes are in the Vega system and [4.5] is the magnitude in the $4.5 \mu \mathrm{m}$ band. The spectroscopically identified galaxies at $z=1.41$ are marked with blue squares, and the photometrically selected galaxies at $1.25<z<1.55$ by red diamonds. The green horizontal line represents a model prediction at $\mathrm{L}^{*}$ for a passively evolving stellar population formed in a single $100 \mathrm{Myr}$ burst beginning at $z_{f}=3.0$, as described in the text. The errors in the photometry are shown only for the spectroscopic member galaxies. The photometry represents $5^{\prime \prime}$ corrected aperture magnitudes. The field size is $3^{\prime} \times 3^{\prime}$.

The scatter seen in the colors of the member galaxies is dominated by the photometric uncertainties in the NDWFS photometry of $I \gtrsim 23$ galaxies.

Also plotted in Figure 5 is an estimate of the expected color for $\mathrm{L}^{*}$ early-type galaxies. Using a simple passive evolution model calculated from the GISSEL of Bruzual \& Charlot (2003) and our assumed cosmology, we cal- 
culated the expected colors for a single 100 Myr burst stellar population formed at $z_{f}=3.0$. As can be seen in Figure 5, the colors of the brighter member galaxies are consistent with those exected for early-type galaxies whose stars formed at $z_{f}=3.0$. The three brightest objects on the color-magnitude relation have not yet been observed spectroscopically.

\section{DISCUSSION}

The observations presented here provide strong evidence supporting the identification of ISCS J143809+341419 as a galaxy cluster at $z=1.41$. Photometric redshifts, demonstrated to be accurate by the optical spectroscopy in the relevant redshift range, indicate a significant overdensity of galaxies within a region of radius $r=300 \mathrm{kpc}$ at $z \sim 1.4$. Our optical spectroscopy shows that 5 of these galaxies are at the same redshift. Further examination of the properties of the cluster, such as its mass and the evolutionary state of its galaxies, is deferred until spectroscopy can confirm more members and deeper multiband imaging can be obtained.

It is noteworthy that ISCS J143809+341419 was detected using imaging taken by IRAC with exposures lasting only 90 s per pointing over the $\sim 9$ degree $^{2}$ area in the Boötes field of the NDWFS. As implied by the identification of another $z \sim 1.4$ cluster which relied on a short $20 \mathrm{ks}$ exposure in the XMM archive (Mullis et al. 2005), current and planned surveys are better off covering larger areas rather than going deep if the goal is to find $z>1$ galaxy clusters. To date three other $z>1$ IRACselected clusters have been spectroscopically confirmed in the Boötes field (P. Eisenhardt et al. in preparation, M. Brodwin et al. in preparation, and R. Elston et al. in preparation). With the combination of the search described here in the Boötes field, serendipitous searches for clusters in the XMM archival data (Romer et al. 2001;
Mullis et al. 2005), and the on-going wide-area surveys such as SWIRE (Lonsdale et al. 2003), the RCS (Gladders \& Yee 2005), and the CFHT-Legacy Survey, the time finally is ripe for the identification of large samples of $z>1$ clusters. The construction of such samples will pave the way towards a better understanding of the origin of early-type galaxies.

This work is based on observations made with the Spitzer Space Telescope, which is operated by the Jet Propulsion Laboratory, California Institute of Technology. The W. M. Keck Observatory is a scientific partnership between the University of California and the California Institute of Technology, made possible by a generous gift of the W. M. Keck Foundation. The authors wish to recognize and acknowledge the very significant cultural role and reverence that the summit of Mauna Kea has always had within the indigenous Hawaiian community; we are most fortunate to have the opportunity to conduct observations from this mountain. The Flamingos Extragalactic Survey and the NOAO Deep Wide-Field Survey would not have been possible without support from NOAO, which is operated by the Association of Universities for Research in Astronomy, Inc., under a cooperative agreement with the National Science Foundation (NSF). We thank the observing teams at the University of Florida and at NOAO for supporting these two surveys. A. H. G. acknowledges support from the NSF AAPF under award AST-0407085 and from an NSF SGER under award AST-0436681. SAS's work was performed under the auspices of the U.S. Department of Energy, National Nuclear Security Administration by the University of California, Lawrence Livermore National Laboratory under contract No. W-7405-Eng-48. We thank the referee for comments leading to an improved version of the paper.

\section{REFERENCES}

Blakeslee, J. et al. 2003, ApJ, 596, L143

Bruzual, G. \& Charlot, S. 2003, MNRAS, 344, 1000

Brodwin, M. 2004, Ph.D. thesis, University of Toronto

Bower, R., Lucey, J.R., \& Ellis, R.S. 1992, MNRAS, 254, 589

Coleman, G.D., Wu, C.-C., \& Weedman, D.W. 1980, ApJS, 43, 393

Conselice, C.J. 2005, astro-ph/0507146

De Propris, R., Stanford, S.A., Eisenhardt, P.R., Dickinson, M., \& Elston, R. 1999, AJ, 118, 719

Eggen, O., Lynden-Bell, D. \& Sandage, A.R. 1962, ApJ, 136, 748

Eisenhardt, P.R. et al. 2004, ApJS, 154, 48

Fazio, G. et al. 2004, ApJS, 154, 10

Gladders, M. \& Yee, H. 2005, ApJS, 157, 1

Januzzi, B. \& Dey, A. 1999, BAAS, 194, 88.03

Kauffmann, G. 1996, MNRAS, 281, 487

Kelson, D., Illingworth, G., van Dokkum, P., \& Franx, M. 2000, ApJ, 531, 184

Kinney, A., Calzetti, D., Bohlin, R.C., McQuade, K., StorchiBergmann, T., \& Henrique, R. 1996, ApJ, 467, 38

Lonsdale et al. 2003, PASP, 115, 897

Lubin, L., Postman, M., Oke, J.B., Ratnatugna, K., Gunn, J.E., Hoessel, J.G., \& Schneider, D.P. 1998, AJ, 116, 584

Mei, S. et al. 2005, ApJ, submitted
Mullis, C. et al. 2005, ApJ, 623, L85

Oke, J.B. et al. 1995, PASP, 107, 3750

Postman, M. et al. 2005, ApJ, 623, 721

Romer, K., Vianna, P.T.P, Liddle, A.R., \& Mann, R.G. 2001, ApJ, 547,594

Rosati, P., Stanford, S.A., Eisenhardt, P.R., Elston, R., Spinrad, H., Stern, D., \& Dey, A. 1999, AJ, 118, 76

Rosati, P. et al. 2004, AJ, 127, 230

Stanford, S.A., Elston, R., Eisenhardt, P.R., Spinrad, H., Stern, D., \& Dey, A. 1997, AJ, 114, 2232

Stanford, S.A., Eisenhardt, P.R., \& Dickinson, M. 1998, ApJ, 492, 461

Stanford, S.A., Holden, B., Rosati, P., Eisenhardt, P., Stern, D., Squires, G., \& Spinrad, H. 2002, AJ, 123, 619

van Dokkum, P., Franx, M., Kelson, D., \& Illingworth, G. 1998, ApJ, 504, L17

van Dokkum, P. \& Franx, M. 2001, ApJ, 553, 90

van Dokkum, P. \& Stanford, S.A. 2003, ApJ, 585, 78

van Dokkum, P. 2005, astro-ph/0506661

Werner, M. et al. 2004, ApJS, 154, 1 
TABLE 1

Summary of Spectroscopic Cluster Members

\begin{tabular}{ccccccc}
\hline \hline ID & R.A. $^{\text {a }}$ & Dec. $^{\text {a }}$ & {$[4.5]$} & phot-z & template & spec-z \\
\hline IRAC J143806+341433 & $14: 38: 06.97$ & $34: 14: 33.8$ & 16.68 & 1.39 & 1 & 1.4153 \\
IRAC J143807+341441 & $14: 38: 07.80$ & $34: 14: 41.6$ & 16.18 & 1.30 & 3 & 1.4166 \\
IRAC J143813+341452 & $14: 38: 13.36$ & $34: 14: 52.9$ & 16.93 & 1.48 & 0 & 1.4028 \\
IRAC J143812+341318 & $14: 38: 12.10$ & $34: 13: 18.2$ & 16.90 & 1.35 & 3 & 1.4147 \\
IRAC J143811+341256 & $14: 38: 11.25$ & $34: 12: 56.2$ & 17.20 & 1.39 & 11 & 1.4172 \\
& & & & & & \\
\hline
\end{tabular}

${ }^{\mathrm{a}}$ Coordinates are J2000. 